\title{
Eventos hirientes, amor compasivo y calidad de vida en parejas con cardiopatía
}

\section{Hurtful events, compassionate love, and quality of life in couples with heart disease}

Luisa Fernanda Granillo Velasco

Rozzana Sánchez Aragón

Universidad Nacional Autónoma de México
German Arturo Gómez Briseño

UCIC Hospital General de México

\section{Resumen}

Debido a que la relación de pareja es un vínculo significativo, es comprensible que cuando uno de los miembros padece una enfermedad cardiaca entren en juego variables positivas y negativas, como los eventos hirientes (EH) y el amor compasivo; los cuales impactan la calidad de vida de la pareja. Por ello, los objetivos fueron: 1) explorar diferencias en la calidad de vida entre personas con cardiopatía y sus parejas, 2) examinar diferencias en la experiencia de EH, el amor compasivo y la calidad de vida en función del sexo, y 3) analizar la relación de la experiencia de EH y el amor compasivo con la calidad de vida. Se trabajó con 204 parejas mexicanas de 18 a 88 años, de los cuales 216 (105 mujeres, 111 hombres) padecían alguna cardiopatía. La batería estuvo conformada por: 1) Escala de Estado de Salud Mental y Física SF-36, 2) Escala de Amor Compasivo y 3) Batería de Eventos Hirientes. Para ello se realizaron pruebas U de Mann-Whitney y análisis de correlación de Spearman. Los resultados muestran diferencias entre la pareja respecto a la salud, mientras que las mujeres indican mayores afectaciones a partir de un EH, así como mayores limitaciones físicas debido a problemas emocionales y de salud. Finalmente, los hallazgos confirman la relación de los EH y el amor compasivo con la calidad de vida, con mayores afectaciones en los pacientes. Estos resultados fueron discutidos según la teoría en donde resalta la interacción de las variables trabajadas en parejas con una enfermedad crónica.

Palabras Clave: pareja, cardiopatía, calidad de vida, eventos hirientes, amor compasivo

Nota del autor

Luisa Fernanda Granillo Velasco, Facultad de Psicología, Universidad Nacional Autónoma de México.

La correspondencia en relación con este artículo debe dirigirse a Luisa Fernanda Granillo Velasco, Universidad Nacional Autónoma de México (UNAM), Circuito Ciudad Universitaria Avenida, Ciudad Universitaria, Ciudad de México, México, C. P. 04510 .

Dirección electrónica: luisagranillovelasco1705@gmail.com 


\begin{abstract}
Since the couple's relationship is a significant link, it is understandable that when one of the members suffers from heart disease, positive and negative variables come into play, such as hurtful events (HE), and compassionate love; affecting the quality of life of the couple. The objectives were: 1) to explore differences in the quality of life between people with heart disease and their partners, 2) to examine differences in the experience of $\mathrm{HE}$, compassionate love and quality of life based on sex, and 3 ) to analyze the relationship of the experience of $\mathrm{HE}$ and compassionate love with quality of life. We worked with 204 Mexican couples between the ages of 18 and 88, of which 216 (105 women, 111 men) suffered from heart disease. The battery consisted of: 1) SF-36 Mental and Physical Health Status Scale, 2) Compassionate Love Scale and 3) Battery of Hurtful Events. For this, Mann-Whitney U tests and Spearman's correlation analysis were performed. The results showed differences in health outcomes between partners, and exhibited that the presence of $\mathrm{HE}$ and compassionate love may be related to the quality of life, which likewise may be more pervasive in people with heart disease. These results were discussed according to the theory, highlighting the interaction of the variables studied in couples with a chronic disease.
\end{abstract}

Keywords: Couple, Heart disease, Quality of life, Hurtful events, Compassionate love

Dentro de las diversas relaciones que el individuo puede entablar a lo largo de su vida, la relación de pareja es una de las más relevantes debido a que en ella se encontrarán expectativas, anhelos $\mathrm{y}$ soportes afectivos $\mathrm{y}$ sociales (Maureira, 2011). Este tipo de relación, estará determinada por ciertas características de cada uno de los miembros que la conforman, por ejemplo, el estilo afectivo, su historia de aprendizaje, el tipo de apego, experiencias anteriores y el contexto en el que se inicia y desarrolla dicha relación (Martínez-Álvarez et al., 2014).

Así, la pareja es una unidad sistemática socio afectiva que incluye un proyecto de vida en común, siendo una etapa de exploración y preparación de acuerdo a ciertas normas (Díaz y Sánchez, 2020), incluyendo además el involucramiento voluntario de los miembros basado en características como la atracción (física y sexual), la personalidad, compatibilidad de intereses, protección, apoyo y reciprocidad. A partir de esto, es comprensible que cuando uno de los miembros de la relación padece una enfermedad crónica como lo es una enfermedad cardiaca, se permee su identidad social, sus roles y planes para el futuro, teniendo entonces un impacto negativo severo (Ledón, 2011) en sus relaciones cercanas y, por supuesto, con su pareja (Dalteg et al., 2011).

Este tipo de enfermedades ubicadas en el grupo de padecimientos con mayor riesgo de mortalidad a nivel mundial (Suárez y Monroy, 2012) son una discapacidad duradera causada por un cambio patológico irreversible que implica tratamiento especial y rehabilitación 
para el paciente, además de cambios y adaptación dentro de aspectos físicos, psicológicos, sociales y ambientales (Ledón, 2011). De esta manera, es la pareja quien tiene la posibilidad de proporcionar mayor apoyo en aspectos que promuevan la salud o amortigüen el estrés dentro de la relación al resultar en un impacto a la calidad de vida de ambos miembros de la relación (Zepeda y Sánchez, 2019).

El surgimiento del término calidad de vida se da a partir de las nociones de salud y bienestar físico, mental y social citados por la Organización Mundial de la Salud; se compone de una valoración subjetiva respecto a la salud que, si bien se basa en los elementos biológicos, estos no son los únicos que afectan la percepción de bienestar o malestar en el individuo (Urzúa, 2010; Burbano, 2017). A partir de esta lógica, se han identificado como los principales factores (Urzúa y Caqueo-Urízar, 2012) a los siguientes: bienestar emocional, riqueza y bienestar material, salud, trabajo y otras formas de actividad productiva, relaciones familiares y sociales, seguridad e integración con la comunidad.

Una derivación del término de calidad de vida se refiere al valor que cada persona asigna a la duración de la vida en función de la percepción de limitaciones físicas, psicológicas y sociales, además de la disminución de oportunidades a causa de un padecimiento (Urzúa, 2010), así como las condiciones y satisfacción con la vida que se establecen a partir de los indicadores antes mencionados y que integrados con los sentimientos de subjetividad respecto a cada área pueden resultar en el bienestar general (Urzúa y Caqueo-Urízar, 2012). Esto se verá afectado por las personas significativas que se encuentran alrededor, como la relación de pareja, la cual resulta fundamental para la calidad de vida de las personas (Dalteg et al., 2011). Y es en ella donde pueden surgir los llamados eventos hirientes (EH), los cuales son definidos como transgresiones, devaluaciones relacionales o actos desfavorables que violan o rompen ciertas reglas acordadas con anterioridad entre la pareja, incluyendo humillación, provocación de celos, indiferencia, amenazas, acusaciones, rechazo, infidelidad, alejamiento o violencia verbal o física - por mencionar algunos - que detonan emociones negativas (Leary, 2015; Vangelisti y Redlick, 2017) y deterioran con ello la calidad de vida así como la relación (Álvarez et al., 2015).

Hay que señalar que el impacto con el que se perciba el evento dependerá de la forma en la que la víctima lo conceptualice y cómo responda a partir de ello (Bachman y Guerrero, 2006). Por ejemplo, Bachman y Guerrero (2006) refieren que en ocasiones las personas pueden considerar la causa de su dolor ante una transgresión o devaluación como fugaz o intrascendente, otras pueden considerar que fue algo grave y provocó un daño irrevocable (Lemay et al., 2012; Vangelisti y Redlick, 2017) y algunas otras pueden sentir que su pareja tuvo realmente la intención de herirle o humillarle. En congruencia, los EH favorecen la percepción 
de que el otro no considera que su relación es tan importante, cercana o valiosa como lo desearía (Leary, 2015); además de que los sentimientos de dolor experimentados evidencian la vulnerabilidad de la persona herida (Vangelisti y Redlick, 2017), por lo que su capacidad para influir y - en ocasiones - destruir la seguridad de una persona (Lemay et al., 2012; Vangelisti y Redlick, 2017) se complementan con su impacto en su salud física y/o psicológica (RuizGonzález et al., 2018).

Por lo tanto, los EH por un lado afectan negativamente a la pareja y a la relación, mientras que por otro otras variables como el amor — particularmente aquel que se caracteriza por su incondicionalidad - pueden generar comportamientos en pro del bienestar de la pareja enferma (Leary, 2015; Sabey et al., 2014). Así, el amor compasivo es una actitud hacia una persona que implica sentimientos, cogniciones y comportamientos centrados en el cuidado, la preocupación, la ternura, y una orientación hacia el apoyo, la ayuda y la comprensión cuando se percibe que sufre o pasa por un momento de necesidad (Sabey et al., 2014).

Quien siente el amor compasivo: 1) tiene la libertad y la voluntad de amar al otro, 2) trata de comprender y aceptar verdaderamente las condiciones y el estado del receptor, 3) le da valía mediante el respeto de su individualidad, 4) comprende la situación en la que se encuentra la persona e identifica lo que podría realmente contribuir a su bienestar, 5) siente un gran compromiso emocional y comprensión para amar plenamente de una manera integrada, 6) es abierto y receptivo ante oportunidades específicas que permitan la expresión de amor compasivo, 7) siente de manera duradera la experiencia de acudir en respuesta al sufrimiento de otra persona para compartir su estado emocional y 8) incluye actitudes y acciones dirigidas al florecimiento del otro con cierto costo para uno mismo tratándose entonces de un sentimiento relacionado a emociones positivas (Fehr et al., 2014; Sabey et al., 2014).

Como puede observarse en la dinámica relacional de la pareja aparecen diversas variables - positivas y negativas - que entran en juego y determinan su propia interacción (Blandón-Hincapié y López-Serna, 2016). Si esto se lleva al contexto de aquellas en las que uno de los miembros padece una cardiopatía, la situación cobra otras dimensiones. Es decir, los EH pueden ser generados por la enfermedad o bien contribuir a malestares físicos y emocionales (Lemay et al., 2012; Vangelisti y Redlick, 2017), y el amor compasivo (Sabey et al., 2014), como antítesis, puede contribuir al cuidado supremo de un ser humano por otro de una manera absoluta y leal; por lo que ambos constructos pueden tener un impacto en la calidad de vida de cada uno de los miembros (Gumán et al., 2015). Por ello, los objetivos de esta investigación fueron: 1) explorar las diferencias en cuanto a la calidad de vida entre las personas con una cardiopatía y sus parejas, 2) examinar las diferencias por sexo en la experiencia de EH, el amor compasivo y la calidad de vida tanto en los pacientes como en sus parejas, y 3) analizar la relación entre la experiencia de $\mathrm{EH}$ y el amor compasivo con la calidad de vida tanto en las personas con cardiopatía como en sus parejas. 


\section{Método}

\section{Participantes}

Se trabajó con una muestra no probabilística por conveniencia (Hernández et al., 2014) cuyos criterios de inclusión eran: ser mayores de edad (18 años), tener escolaridad mínima de secundaria, tener pareja con la cual se cohabitara y participara en la investigación, y que alguno de los miembros padeciera una cardiopatía y el otro se identificara como sano. A partir de esto se obtuvo un total de 204 (50\% mujeres y $50 \%$ hombres) parejas heterosexuales residentes de la Ciudad de México, cuyas edades oscilaron de 18 a 88 años $(M=74.60$, Moda $=45, D E=11.68)$ y de los cuales $216(105$ mujeres y 111 hombres) participantes padecían alguna cardiopatía. En términos de escolaridad, 105 contaban con estudios de secundaria, 130 con preparatoria y 170 con licenciatura (tres no respondieron). Respecto a su estado civil, 100 de ellos vivían en unión libre y 308 casados, con un tiempo de relación de 10 meses a 50 años $(M$ $=22.32, D E=142.83)$. Del total de la muestra, 64 de los participantes indicaron no tener hijos, mientras que el resto reportó tener de uno a ocho hijos $(M=1.92, D E=1.264)$.

\section{Instrumentos}

Para el cumplimiento de los objetivos se aplicaron los siguientes instrumentos (Tabla 1).

\section{Tabla 1}

Confiabilidad de los instrumentos utilizados en el presente estudio

\begin{tabular}{clc}
\hline \multicolumn{1}{c}{ Instrumento } & \multicolumn{1}{c}{ Factor } & Alpha de Cronbach $(\alpha)$ \\
\hline & Rechazo & 0.74 \\
Eventos Hirientes & Intención & 0.72 \\
& Autopercepción positiva & 0.69 \\
& Autopercepción negativa & 0.88 \\
Amor Compasivo & Efecto en sí mismo & 0.84 \\
& Efecto en la relación & 0.88 \\
& Apoyo emocional y empatía & 0.88 \\
& Amor altruista & 0.79 \\
Calidad de Vida & Función física & 0.88 \\
& Rol físico & 0.89 \\
& Rol emocional y función Social & 0.91 \\
& Vitalidad & 0.90 \\
& Dolor & 0.84 \\
& Salud en general & 0.78 \\
\hline
\end{tabular}


Escala de Estado de Salud Mental y Física SF36 (Sánchez et al., 2017; Ware y Sherbourne, 1992)

Para la presente investigación fue utilizada la versión validada en México, la cual consta de 44 reactivos en formato de respuesta tipo Likert de cinco opciones (1-nada a 5-mucho) y que se encuentran distribuidos en dos secciones, $y$ además dos indicadores de salud en general y en comparación con el año pasado $(\alpha=0.73)$. La primera sección consiste en 40 reactivos pertenecientes a cinco factores $(\alpha=0.76$ a 0.95$)$ y que explicaron el $65.36 \%$ de la varianza: 1) Rol emocional y función social (p.ej. "puso menos atención a la hora de estar realizando su trabajo o actividades cotidianas debido a algún problema emocional como estar triste, deprimido o nervioso"), 2) Función física (p.ej. "le cansa caminar varias manzanas o centenares de metros"), 3) Rol físico (p.ej. "tuvo que dejar de hacer algunas tareas en su trabajo o en sus actividades cotidianas a causa de su salud física"), 4) Dolor corporal (p.ej. "tuvo dolor en alguna parte del cuerpo"), 5) Vitalidad (p.ej. “¿qué tanto se sintió calmado y tranquilo?”); y la segunda sección compuesta por 4 reactivos en un solo factor que explicó el $55 \%$ de la varianza, nombrado Salud en general (p.ej. "estoy tan sano como cualquiera", "mi salud es excelente").

\section{Escala de Amor Compasivo (Flórez y Sánchez,} 2019)

Conformada por 33 reactivos $(\alpha=.973)$ con formato de respuesta tipo Likert de cinco opciones que indican grados de acuerdo distribuidos en 2 factores que explicaron el $61.96 \%$ de la varianza: 1) Apoyo emocional y empatía ( $\alpha=0.96)$, con reactivos como "trato de entender más que juzgar a mi pareja", "me mueve emocionalmente cuando mi pareja está estresada" y "cuando veo a mi pareja triste, siento necesidad de ayudarle", y 2) Amor altruista ( $\alpha=$ 0.92 ), compuesto por reactivos como: "prefiero comprometerme en acciones que ayuden a mi pareja", "tiendo a sentir compasión por mi pareja" y "hago lo que sea necesario para ser de ayuda a mi pareja".

\section{Batería de Eventos Hirientes (Pérez y Sánchez,} 2016)

Consta de una pregunta de selección múltiple relativa a haber experimentado alguna situación con su pareja donde la persona se sintiera herida u ofendida, con las siguientes opciones de respuesta: celos, crítica, indiferencia, infidelidad, decir que ya no le quiere, violencia verbal o física u otra situación $\mathrm{u}$ otra. Asimismo, presenta tres subescalas cuyos reactivos en formato tipo Likert de cinco opciones de respuesta que indican grados de acuerdo se presentan a continuación.

\section{1) Reacciones emocionales ( 7 reactivos).} Agrupa cuatro indicadores que explicaron en conjunto el $62.79 \%$ de la varianza total y con un $\alpha=0.83$ : a) Daño (“¿cuán herido se sintió?”), b) Rechazo ("¿cuán rechazado por su pareja se sintió?”, “¿cuán repudiado por su pareja se sintió?”), c) Control (“¿cuánto control sintió que tuvo sobre la situación, en comparación con su pareja?”, “¿cuánto poder le faltó en la situación, en comparación con su pareja?”) y d) 
Intencionalidad “ ¿usted cree que su pareja tenía la intención de herirlo/lastimarlo?”, “¿usted cree que su pareja sabía que lo había herido/ lastimado?").

2) Autopercepciones (10 reactivos). Agrupados en dos factores explican el $52.9 \%$ de la varianza; comprende dos factores: a) Positivas (con adjetivos como atractivo, inteligente) ( $\alpha=$ $0.91)$ y b) Negativas (con atributos como tonto, incompetente) ( $\alpha=0.92)$.

\section{3) Efectos de los EH (8 reactivos).}

Agrupados en dos factores explicaron el 57.29

$\%$ de la varianza total: a) Efectos sobre sí mismo ("me hizo menos seguro en situaciones similares en las que mis sentimientos fueron heridos") con $\alpha=0.89$ y b) Efectos sobre la relación ("esto hizo que la relación con mi pareja se debilitara temporalmente") con $\alpha=0.87$.

\section{Tipo de estudio}

Este es un estudio — en primer momentode tipo comparativo (Cozby, 2005) debido aque se examinan las diferencias entre distintos grupos en función de tener una cardiopatía y no tenerla y el sexo en las variables examinadas. Además se considera correlacional (Hernández et al., 2014), al explorarse el grado de asociación entre la experiencia de EH, el amor compasivo y la calidad de vida en personas con una cardiopatía y sus parejas.

\section{Procedimiento}

Los instrumentos fueron aplicados por cuatro psicólogas entrenadas, en lugares públicos (escuelas, parques, hospitales, clínicas, etc.) donde se solicitaba verbalmente la participación voluntaria, confidencial y anónima de personas que cumplieran con los criterios de inclusión. Quienes desearon participar respondieron una batería de pruebas, se resolvieron dudas durante la aplicación y se pusieron a disposición de las personas los resultados en caso de desearlo.

\section{Consideraciones éticas}

Con base en el artículo 17 del Reglamento de la Ley General de Salud en Materia de Investigación para la Salud, la investigación presente es considerada de riesgo mínimo al tratarse de la aplicación de pruebas psicológicas en las que no se manipula la conducta de los participantes, únicamente se realizan las preguntas necesarias para la instigación y se evita indagar en aspectos personales que no contribuyan estadística y científicamente. Asimismo, se realizó un consentimiento informado de manera verbal en el cual los participantes eran conscientes de que sus datos únicamente serían utilizados para el desarrollo de la ciencia y que podían sentirse libres de abandonar el estudio en cualquier momento sin ninguna represalia. Con base en esto, todo procedimiento se realizó bajo el Código Ético del Psicólogo (Sociedad Mexicana de Psicología, 2010) y la Asociación Americana de Psicología (2017).

\section{Análisis de datos}

Respecto al procedimiento para elanálisis de datos se utilizó el programa estadístico SPSS Statistics 21. En un primer momento se realizó una prueba de normalidad dirigido a comprobar el cumplimiento de supuesto de normalidad (Kolmogorov-Smirnov) de las variables estudiadas en este caso en la muestra 
en cuestión. Dado que este principio no se cumplió, se utilizó un estadístico no paramétrico rechazándose la hipótesis nula que indica homogeneidad en la muestra. Posteriormente y en busca de cubrir el primer objetivo dirigido a explorar diferencias en calidad de vida entre los pacientes y sus parejas sanas, se realizó una prueba U de Mann-Whitney considerándose dos grupos: Grupo 1, conformado por 216 personas del total de la muestra y que padecían alguna cardiopatía, y el Grupo 2, compuesto por 192 personas sanas. A continuación, se realizó igualmente una prueba $U$ de Mann-Whitney para examinar las diferencias en la experiencia de EH, el amor compasivo y la calidad de vida en función del sexo en los grupos ya mencionados. Finalmente, se realizó un análisis de correlación de Spearman, por un lado, en los pacientes con cardiopatía y, por otro, en las personas sanas.

\section{Resultados}

\section{Diferencias en calidad de vida en función de la enfermedad}

A partir de la prueba U de Mann-Whitney resulta que quienes padecen alguna enfermedad clasificada como cardiopatía refirieren mayores limitaciones al hacer esfuerzos físicos diversos como mover una mesa, subir las escaleras, agacharse o arrodillarse (Función física). Además reportan - en mayor media-haber hecho menos trabajo, actividades y tareas cotidianas, así como actividades sociales de las deseadas debido a su salud física (Función física) y problemas emocionales (Rol emocional y función social), además de mayor dolor, debilidad y cansancio (Dolor). Todo esto en comparación con sus parejas sanas, en quienes además se observa reportan mayormente una buena salud, salud en general y vitalidad (Tabla 2).

\section{Tabla 2}

Diferencias entre pacientes y sus parejas en calidad de vida

\begin{tabular}{|c|c|c|c|c|}
\hline Factor & $\begin{array}{c}\text { Personas } \\
\text { con cardiopatía }\end{array}$ & $\begin{array}{c}\text { Parejas } \\
\text { sanas }\end{array}$ & $\mathrm{p}$ & PS \\
\hline Función física & 237.32 & 159.85 & 0.00 & 0.30 \\
\hline Rol físico & 239.81 & 155.32 & 0.00 & 0.28 \\
\hline Rol emocional y función social & 221.62 & 184.07 & 0.01 & 0.40 \\
\hline Vitalidad & 185.40 & 210.31 & 0.00 & 0.43 \\
\hline Dolor & 223.32 & 174.09 & 0.00 & 0.37 \\
\hline Salud en general & 191.19 & 216.23 & 0.01 & 0.43 \\
\hline En general, usted diría que su salud es: & 159.38 & 237.21 & 0.00 & 0.30 \\
\hline
\end{tabular}

${ }^{*} \mathrm{p}<0.05$ 
Diferencias en la experiencia de EH, amor compasivo y calidad de vida en función del sexo en pacientes con cardiopatía y sus parejas sanas

En lo correspondiente a los pacientes, respecto a la experiencia de $\mathrm{EH}$, se observan similitudes entre sexos en cuanto al rechazo, control ante la situación, intención percibida del otro y autopercepción positiva posterior a una transgresión. De la misma forma, para el amor compasivo las similitudes son referentes al amor altruista mediante conductas como preferir ayudar a la pareja y sentir compasión por ella. Mientras que, para la calidad de vida en estos mismos participantes, los resultados son similares entre hombres y mujeres respecto a las limitaciones al realizar actividades diversas debido a problemas de salud (Rol físico), vitalidad y dolor.
Sin embargo, se observan diferencias significativas en cuanto a que las mujeres indican una mayor autopercepción negativa como sentirse tontas e indeseables, mayor impacto personal con decaimiento de su autoestima y seguridad, y mayores afectaciones en la relación a partir de su debilitamiento temporalo permanente posterior a la experiencia de unEH. Además, en el amor compasivo, los resultados indican que los hombres muestran mayores conductas de apoyo, ánimo y cuidado a su pareja (Apoyo emocional y empatía). Finalmente en la calidad de vida, son las mujeres quienes indican mayores limitaciones en sus actividades diarias y sociales debido a problemas emocionales (Rol emocional) en comparación con los hombres que reportan mayor salud en general (Tabla 3).

\section{Tabla 3}

Diferencias en EH, amor compasivo y calidad de vida en los pacientes con cardiopatía en función del sexo

\begin{tabular}{llcccc}
\hline & \multicolumn{1}{c}{ Factor } & Mujer & Hombre & p & PS \\
\hline Eventos Hirientes & Autopercepción negativa & $\mathbf{1 0 5 . 1 2}$ & 86.47 & 0.01 & 0.40 \\
& Efecto en sí mismo & $\mathbf{1 1 0 . 6 1}$ & 85.77 & 0.00 & 0.37 \\
& Efecto en la relación & $\mathbf{1 1 4 . 6 5}$ & 83.31 & 0.00 & 0.34 \\
Amor Compasivo & Apoyo emocional y empatía & 93.92 & $\mathbf{1 2 0 . 5 8}$ & 0.00 & 0.37 \\
Calidad de Vida & Función física & $\mathbf{1 1 7 . 1 4}$ & 97.32 & 0.01 & 0.40 \\
& Rol emocional y función social & $\mathbf{1 2 1 . 3 3}$ & 96.36 & 0.00 & 0.38 \\
& Salud en general & 96.47 & $\mathbf{1 1 7 . 9 3}$ & 0.01 & 0.39 \\
\hline
\end{tabular}

${ }^{*} p<0.05$ 
Por otro lado, respecto a las diferencias por sexo en las parejas de los pacientes, los resultados indican similitudes respecto a la calidad de vida, el amor compasivo, tanto en el apoyo emocional y empatía como en el amor altruista con la pareja, y en la experiencia de
EH. En esta última variable, las diferencias significativas se observan únicamente en la percepción de que dicho evento impactó la relación al provocar su debilitamiento temporal o permanente y desconfianza entre sus miembros (Efecto en la relación), en donde son las mujeres quienes puntúan más alto (ver Tabla 4).

\section{Tabla 4}

Diferencias en EH, amor compasivo y calidad de vida en las parejas de los pacientes en función del sexo

\begin{tabular}{lccccc}
\hline & Factor & Mujer & Hombre & $\mathrm{p}$ & PS \\
\hline Eventos Hirientes & Efecto en la relación & 90.86 & 75.04 & 0.03 & 0.40 \\
\hline$* \mathrm{p}<0.05$ & & & & &
\end{tabular}

Para continuar con el cumplimiento de los objetivos, se realizó un análisis de correlación de Spearman dirigido a analizar la relación de la experiencia de EH y el amor compasivo con la calidad de vida, tomando en consideración el sexo. A partir de ello se observó que, en las personas con cardiopatía -únicamente en los hombres - a más sentimientos de rechazo ante la ocurrencia de un EH, más dolor físico, sentimiento de debilidad y cansancio (Dolor), se tienen mayores limitaciones para realizar diversas actividades cotidianas y sociales a causa de problemas emocionales (Rol emocional y función social) y de salud (Rol físico), asícomo mayores limitaciones al realizar actividades como subir escaleras, agacharse o caminar varias manzanas (Función física). Por otra parte, a mayor percepción de intención de hacer daño por parte de la pareja, menor salud y vitalidad.
Contrario a esto, resultó que a más autopercepción positiva como sentirse atractivo e inteligente aún después de la transgresión, más percepción de salud. Respecto a la autopercepción negativa se observó, solo en los hombres, que sentirse más tonto, indeseable y ridículo ante un EH se relacionó con mayor dolor, más limitaciones con diversas actividades debido a problemas de salud y menor salud percibida. Además, a mayor sensación de que el evento sucedido provocó un efecto personal como decaimiento de la autoestima, inseguridad y preocupación de ser herido nuevamente (Efecto en sí mismo), más problemas de salud que limitan la realización de diversas actividades, más dolor, y menor salud y vitalidad.

Finalmente, en estos mismos participantes se observó que quienes refieren sentir que el EH debilitó la relación ya sea temporal 
o permanentemente creando, además, desconfianza en la pareja en mayor medida (Efecto en la relación) reportan mayores limitaciones al realizar su trabajo o actividades cotidianas sociales debido a problemas emocionales y en su salud física, mayores dolores corporales y menor salud y vitalidad. Por otra parte, en las mujeres con cardiopatía resultó que a mayores sentimientos de control ante la situación en comparación con su pareja ante la experiencia de un $\mathrm{EH}$, menos problemas emocionales que las limiten a realizar actividades

cotidianas y sociales (Rol emocional y función social). Ocurrió de igual manera para ambos sexos cuando se reporta mayor desconfianza y percepción de debilitamiento de la relación ante una transgresión,

Respecto a la relación del amor compasivo con la calidad de vida, se observó — solamente en las mujeres - que expresar más sentimientos de apoyo, ayuda, ánimo y cuidado a la pareja (Apoyo emocional y empatía), se relacionó con mayor salud en general reportada (Tabla 5).

\section{Tabla 5}

Correlaciones de EH y Amor Compasivo con Calidad de Vida en pacientes con cardiopatía por sexo

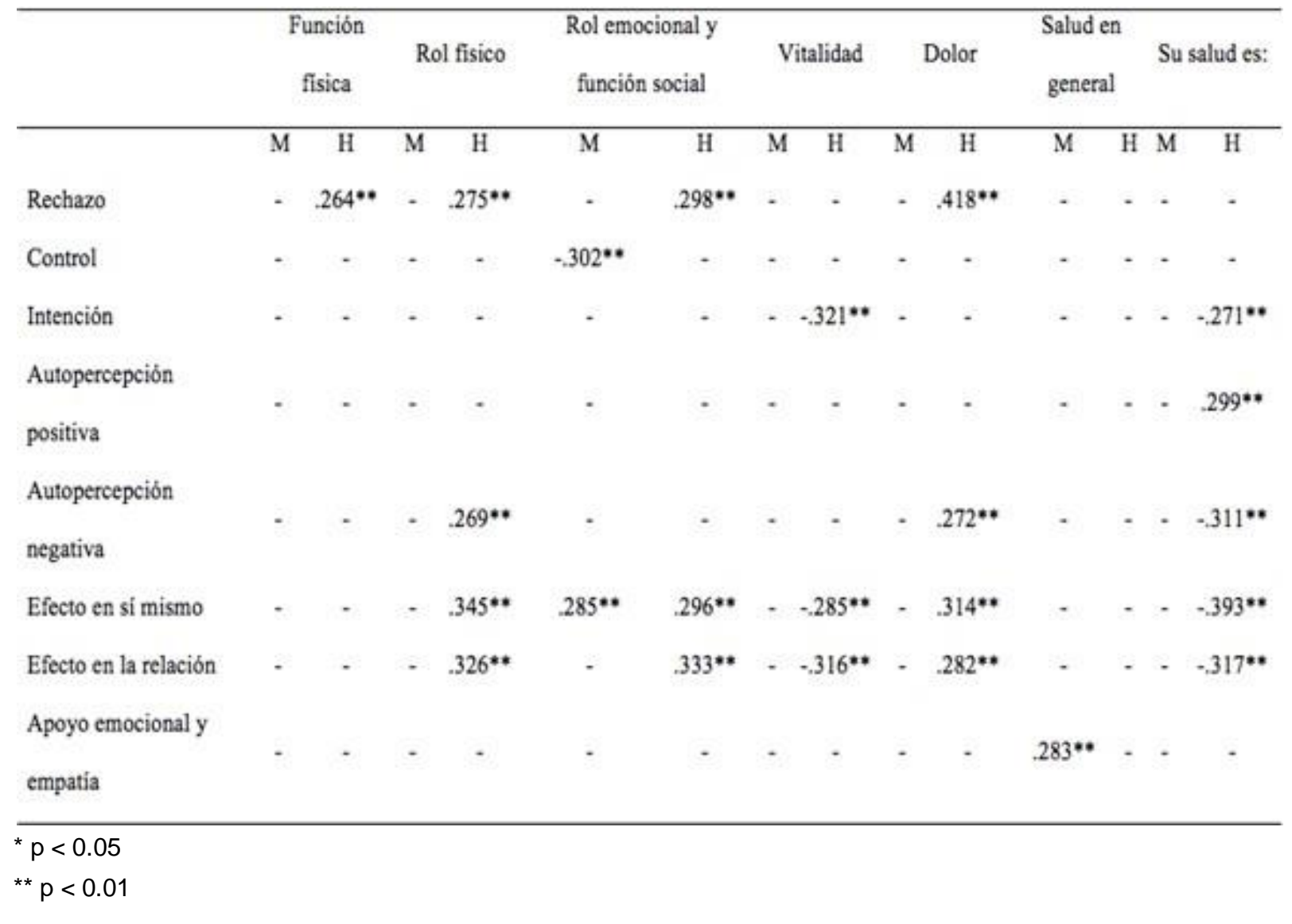


Finalmente, a partir de un análisis de correlación de Spearman, se encontró respecto a las parejas de las personas con cardiopatía, es decir, las personas sanas, que en los hombres experimentar mayor control en comparación con la pareja ante un EH se relacionó con menores problemas emocionales que limiten la realización de actividades diarias y sociales (Rol emocional y función social). Además, en estos mismos participantes se observó que a mayor intención percibida de hacer daño a partir de la transgresión, mayor dolor y menor salud y vitalidad. Mientras que mayor autopercepción negativa se relacionó con mayores dolores corporales y debilitamiento. Ocurrió esto último también cuando los hombres perciben que el evento impactó en la relación al ocasionar su debilitamiento y desconfianza entre sus miembros (Efecto en la relación), aunado a mayores limitaciones en actividades cotidianas debido a problemas de salud (Rol físico). Por otro lado, en las mujeres sanas se observó que a mayores efectos percibidos en la relación a partir de la ocurrencia de un EH, mayores limitaciones al realizar actividades diarias y de trabajo debido a problemas emocionales y mayor dolor. También se encontró que mayor autopercepción negativa posterior a la transgresión se relacionó con menores sentimientos de vitalidad $y$ felicidad.

Respecto a ambos sexos, resultó que percibir mayores sentimientos de inseguridad y decaimiento de la autoestima ante un $\mathrm{EH}$ (Autopercepción Negativa) se relacionó con mayores limitaciones al realizar actividades cotidianas y sociales debido a problemas de tipo emocional (Rol Emocional) y menor vitalidad. Sucedió también esto último cuando el efecto del evento se percibe en la relación a partir de su debilitamiento, así como al crear desconfianza entre sus miembros (Efecto en la relación).

\section{Tabla 6}

Correlación de EH y Amor Compasivo con Salud en parejas de los pacientes con cardiopatía (sanos) por sexo

\begin{tabular}{|c|c|c|c|c|c|c|c|c|c|c|}
\hline & \multicolumn{2}{|c|}{ Rol físico } & \multicolumn{2}{|c|}{$\begin{array}{l}\text { Rol emocional } \\
\text { y función social }\end{array}$} & \multicolumn{2}{|c|}{ Vitalidad } & \multicolumn{2}{|c|}{ Dolor } & \multicolumn{2}{|c|}{ Su salud es } \\
\hline & M & $\mathrm{H}$ & $\mathrm{M}$ & $\mathrm{H}$ & $M$ & $\mathrm{H}$ & $M$ & $\mathrm{H}$ & $\mathrm{M}$ & $\mathrm{H}$ \\
\hline Control & - & - & - & $-.318^{* *}$ & - & - & - & - & - & - \\
\hline Intención & - & - & - & - & - & $-.311^{* *}$ & - & $.319^{* *}$ & - & $-.336^{* *}$ \\
\hline $\begin{array}{l}\text { Autopercepción nega- } \\
\text { tiva }\end{array}$ & - & - & - & - & $-.329^{\star \star}$ & - & - & $.333^{\star *}$ & - & - \\
\hline Efecto en sí mismo & - & $.329^{* *}$ & $.321^{* *}$ & $.383^{* *}$ & $-.308^{* *}$ & $-.440^{\star \star}$ & - & $.398^{\star *}$ & - & - \\
\hline Efecto en la relación & - & - & $.434^{\star *}$ & - & $-.484^{\star *}$ & $-.370^{\star *}$ & $.303^{* *}$ & - & - & - \\
\hline Amor altruista & - & - & - & - & $.226^{\star *}$ & $.403^{\star *}$ & $.304^{* *}$ & - & - & - \\
\hline
\end{tabular}


Por último, en lo correspondiente al amor compasivo y la calidad de vida, resultó que solo en las mujeres sanas - estar más dispuestas a sacrificarse y preocuparse más por el bienestar de su pareja (Amor altruista) se relacionó con mayor dolor corporal. Mientras que tanto en hombres como en mujeres sanos se encontró que, a más amor altruista, mayor vitalidad y felicidad (Tabla 6).

\section{Discusión}

La presente investigación se dirigió hacia tres objetivos: 1) explorar las diferencias en calidad de vida entre las personas con una cardiopatía y sus parejas sanas; 2) examinar las diferencias por sexo en la experiencia de EH, el amor compasivo y la calidad de vida tanto en los pacientes como en sus parejas, y 3) analizar la relación entre la experiencia de $\mathrm{EH}$, el amor compasivo y la calidad de vida en ambos grupos.

Respecto al primer objetivo, se encontró que quienes padecen algún padecimiento clasificado como cardiopatía refirieron mayores limitaciones al hacer actividades que requieran diferentes niveles de esfuerzo físico como subir escaleras, agacharse o mover una mesa, así como haber hecho - en mayor medida - menos trabajos, actividades y tareas cotidianas, y actividades sociales de las deseadas debido a su salud física y problemas emocionales. También refirieron más dolor, debilidad y cansancio, en comparación con sus parejas sanas, quienes reportaron mejor salud, más salud en general y vitalidad.

En congruencia con lo anterior, Castañeda (2015) explica que una enfermedad crónica puede afectar en diferentes dimensiones, al generarle síntomas de diversa índole que puedan causarle deterioro respecto a su funcionalidad social, física y psicológica (Barrantes, 2010; Ledón, 2011) que resultan en un aumento de necesidades de apoyo y soporte. En el mismo tenor, se ha encontrado que dichos pacientes experimentan limitaciones para realizar actividades básicas cotidianas, así como afectaciones emocionales que suponen diversos factores negativos en su calidad de vida en relación con el contexto cultural, sus metas, objetivos, expectativas, valores y preocupaciones propias (Jasso-Soto et al., 2017; Vinaccia-Alpi y Quiceno, 2012).

\section{Diferencias en el paciente con cardiopatía}

Para el cumplimiento del segundo objetivo, referente a las diferencias por sexo en las personas enfermas, resultaron similitudes en cuanto a la experiencia de $\mathrm{EH}$, es decir, hombres y mujeres indicaron de igual manerael rechazo percibido, la sensación de control ante la situación y la intención percibida, así como sentirse atractivos, deseables e inteligentes ante el evento negativo. Estos hallazgos pueden atribuirse a lo mencionado por Vangelisti y Redlick (2017), Mao-Chia (2017) y Lemay et al. (2012), quienes mencionan que, más que con el sexo, la experiencia de transgresiones, devaluaciones o mensajes hirientes dentro de las relaciones y los sentimientos heridos que estos generan, están relacionados en mayor medida con indicadores de tipo emocional y conductual, es decir, las víctimas percibirán el daño según las actitudes y respuestas de los perpetradores, así como las consecuencias percibidas, el 
compromiso, la dependencia y vulnerabilidad de cada persona.

En el amor compasivo las similitudes fueron en cuanto al amor altruista, resultó que los participantes de ambos sexos reportaron ayudar preferencialmente a su pareja y sentir compasión por ella de igual manera. Esto puede ser debido a que, como lo explican Díaz y Sánchez (2020), la relación de pareja es un vínculo significativo y emocional dentro del cual destacan elementos como la afectividad (Sánchez, 2018), el apoyo (Zepeda y Sánchez, 2019) y el amor incondicional mediante la presencia de sentimientos, cogniciones y comportamientos centrados en el cuidado, la preocupación y comprensión en momentos de necesidad (Berscheid, 2010; Sabey et $a l ., 2014)$. Asimismo, al tratarse de conductas en beneficio y florecimiento del otro, las personas - hombres y mujeres - pueden buscar la expresión del amor compasivo en busca de favorecer las emociones positivas dentro de la relación y construir un vínculo saludable (Sabey et al., 2014; McCoy et al., 2017).

También se encontraron similitudes respecto a la calidad de vida en las limitaciones al realizar diversas actividades cotidianas debido a problemas de salud, en el sentimiento de vitalidad y los dolores corporales. Tomando en consideración que se trata de personas con una enfermedad crónica como lo es una cardiopatía, es comprensible que, en general, todas las personas experimenten en diversos grados cambios físicos, psicológicos, sociales y ambientales, los cuales permeen las diferentes esferas de su calidad de vida (Dalteg et al., 2011; Jasso-Soto et al., 2017; Ledón, 2011).
Sin embargo, resultaron diferencias significativas en cuanto a que las mujeres reportaron - en mayor medida- percibirse negativamente sintiéndose tontas e indeseables, decaimiento de su autoestima e inseguridad, así como debilitamiento de su relación de pareja ante la ocurrencia de un EH. Congruente a esto, Lemay et al. (2012) y Vangelisti y Redlick (2017) mencionan que los $\mathrm{EH}$ tienen la capacidad para impactar en la seguridad de la víctima, al generar afectaciones personales dependiendo de factores emocionales como el compromiso, la dependencia y la vulnerabilidad (Lemay et $a l ., 2012$ ). Aunado a esto, se ha encontrado que culturalmente las mujeres son más expresivas en comparación con los hombres y buscan cumplir con rasgos expresivos socialmente aceptados por los otros como el compromiso y preocupación por los sentimientos propios y del otro, además, logran reconocer, admitir y expresar más fácilmente sus emociones (Díaz, Velasco, y Rivera, 2018; Machín y Cifre, 2020), por lo cual experimentan mayores afectaciones psicológicas y ambientales cuando especialmente de su pareja se trata (HamamaRaz, 2010).

En lo correspondiente al amor compasivo fueron los hombres quienes mostraron brindar mayor apoyo, ánimo y cuidado a su pareja en comparación con las mujeres. Dicho hallazgo concuerda con la investigación realizada por McCoy et al. (2017) en donde los hombres informaron igualmente mayor amor altruista hacia su pareja. De la misma manera, Aguilar et al. (2013) indican que los hombres señalan en mayor medida tomar un papel protector, de 
ayuda y cuidado asociados con un perfil más instrumental (Díaz et al., 2018).

Respecto a la calidad de vida, fueron las mujeres quienes indicaron mayores limitaciones en sus actividades diarias y sociales debido a problemas emocionales en comparación con los hombres, quienes reportaron mayor salud en general. Asimismo, como ya se mencionó, las mujeres tienen un perfil más expresivo y por ello sus emociones pueden verse relacionadas con diferentes aspectos de su vida (Díaz et al., 2018; Machín y Cifre, 2020) con lo que pueden percibir un mayor impacto en su salud debido a dicha susceptibilidad psicológica, aunado a su papel dentro de la sociedad con mayores cargas y presiones asociadas a los diferentes roles como ser madre, hija, pareja y empleada, que desempeñan en su día a día (Hamama-Raz, 2010).

\section{Diferencias en la pareja del paciente (sano)}

Respecto a las diferencias por sexo en las parejas de los pacientes, los resultados indicaron similitudes respecto a la calidad de vida en general. Como se ha mencionado, esto puede atribuirse a que, de manera general, una enfermedad cardiaca representa un impacto a la calidad de vida y bienestar de los pacientes en menor o mayor grado (Ledón, 2011), según el conocimiento del diagnóstico, la cantidad de modificaciones experimentadas en la vida diaria, el tratamiento y sus posibles efectos secundarios, la cronicidad del padecimiento y las enfermedades asociadas (Burbano, 2017). Así, aunque el sexo puede ser relevante como factor de riesgo en el padecimiento cardiaco, puede pasar a segundo plano cuando la enfermedad ya se padece y se anteponen los elementos relacionados con las diferentes esferas de la vida del enfermo (familiar, de pareja, laboral) así como con sus limitaciones físicas y psicológicas (Ledón, 2011; Urzúa, 2010).

Mientras que, para el amor compasivo, tanto hombres como mujeres indicaron igualmente brindar apoyo, cuidado y ánimo a su pareja, así como sacrificarse y sentir compasión por ella. En congruencia, Fehr et al. (2014), McCoy et al. (2017) y Sabey et al. (2014) hacen referencia a que el amor compasivo ha sido relacionado positivamente con el funcionamiento de la pareja favoreciendo comportamientos prosociales como el cuidado y el apoyo de sus miembros. Además, se han identificado diversos factores asociados con el desarrollo de este tipo de amor dentro de las relaciones cercanas como la salud, el sexo y los estilos de apego y amor (Fehr et al., 2014; McCoy et al., 2017). Sin embargo, las parejas que se enfrentan a problemas de salud pueden brindar oportunidades para que los cónyuges sientan el apoyo y preocupación del otro ante los desafíos presentes (Carr et al., 2014; McCoy et al., 2017), lo cual resulta en que indiquen de manera similar el apoyo y amor compasivo por su pareja (Fehr et al., 2014; McCoy et al., 2017).

En cuanto a los $\mathrm{EH}$, se observaron similitudes respecto al sentimiento de rechazo, percepción de mayor control ante la situación, intención percibida, autopercepción tanto positiva como negativa y afectaciones personales 
ante la experiencia de una transgresión. En congruencia con lo discutido respecto a los pacientes, la experiencia de EH dentro de la pareja y los sentimientos negativos que se dan a partir de estos han sido relacionados -en su mayoría - con indicadores emocionales y conductuales al momento de la transgresión y las respuestas proporcionadas posteriormente (Vangelisti y Redlick, 2017; Mao-Chia, 2017; Lemay et al., 2012). Sin embargo, las diferencias encontradas en cuanto a que las mujeres reportaron mayores efectos percibidos en la relación al experimentarse debilitamiento temporal o permanente y desconfianza entre sus miembros, pueden explicarse con base en Díaz et al. (2018) y Machín y Cifre (2020) quienes indican que social y culturalmente son las mujeres quienes se han caracterizado por ser más expresivas y con ello lograr admitir y reconocer sus emociones ante diversos eventos con más facilidad en comparación con los hombres

\section{El paciente con cardiopatía}

Ahora, en lo correspondiente al tercer objetivo encaminado a examinar la relación entre las variables trabajadas tanto en los pacientes como en sus parejas sanas, resultó que, respecto a la experiencia de eventos hirientes y la calidad de vida en las personas con cardiopatía —únicamente en los hombres-, a más sentimientos de rechazo ante la ocurrencia de un EH, más dolor físico, debilidad y cansancio se reportan, mayores limitaciones para realizar diversas actividades cotidianas y sociales a causa de problemas emocionales y de salud, así como mayores limitaciones al realizar actividades como subir escaleras, agacharse o caminar varias manzanas. Asimismo, respecto a una mayor intención percibida ante la transgresión ocurrida, los participantes reportaron menor salud y vitalidad.

Respecto a esto, Castañeda (2015) y Urzúa (2010) relatan que las personas con un padecimiento crónico experimentan diversos cambios tanto físicos como psicológicos, además de reacciones emocionales como ansiedad, depresión o tristeza (Montalvo-Prieto et al., 2012), lo cual deja al paciente con cierta vulnerabilidad ante situaciones difíciles como lo puede ser la ocurrencia de un EH. Este, a su vez, es generador de sentimientos heridos que se expresan a través de diferentes emociones negativas (Granillo y Sánchez, 2020; Vangelisti y Redlick, 2017) teniendo como resultado implicaciones importantes para la salud física como agotamiento de los recursos físicos y sociales, o alteraciones en algunas funciones debido a problemas emocionales. Aunado a esto, el sentimiento de rechazo es uno de los que más se asocian con el desánimo, el cual, se exterioriza físicamente debido a su relación con diversos sistemas fisiológicos (Montalvo-Prieto et al., 2012), mientras que la intencionalidad puede influir poderosamente en dicha percepción ante la ocurrencia de un evento de este tipo (Lemay et al., 2012; Vangelisti y Redlick, 2017).

En contraste con lo anterior, se encontró que en los hombres con cardiopatía, una mayor autopercepción positiva como sentirse atractivo e inteligente aún después de la transgresión se relacionó con más percepción de salud. Al respecto, se ha encontrado que la percepción 
positiva de uno mismo es un componente de la personalidad positiva que provee factores de protección y potenciadores de conductas saludables (Flórez et al., 2005), relacionado con los procesos de resistencia y fortaleza ante situaciones difíciles y de adaptación. Es decir, la personalidad positiva supondrá un afrontamiento ante estímulos o eventos aversivos y estresantes, haciendo más fácil el control de dichas situaciones mediante un ajuste de demandas del entorno y expectativas personales (Flórez et al., 2005; Moreno et al., 2005).

Por el contrario, se observó, solo en los hombres, que sentirse más tonto, indeseable y ridículo, así como percibir que la transgresión impactó de manera tanto personal como relacional al provocar inseguridad, debilitamiento de la pareja y desconfianza entre sus miembros ante el $\mathrm{EH}$, se relacionó con mayores limitaciones con diversas actividades debido a problemas emocionales y de salud, más dolor y menor salud percibida, así como menor vitalidad cuando de los efectos del EH se trata. Asimismo, como ya se ha mencionado a lo largo de la presente investigación, la experiencia de un $\mathrm{EH}$ puede tener consecuencias negativas de diversa magnitud según la intencionalidad, la percepción de rechazo, la autopercepción, entre otros, de modo que una transgresión puede tener la capacidad para destruir la seguridad de la víctima (Vengelisti y Redlick, 2017).

En relación con esto, la autoestima y la manera de percibirse a sí mismo han sido elementos asociados con diversas estrategias conductuales de salud (Ruiz-González et al.,
2018), de modo que una alta autoestima se relacione con seguridad, felicidad y satisfacción con la vida, mientras que una baja en la autoestima se vincule, en mayor medida, con deficiencias personales (inseguridad, vulnerabilidad) y patológicas (Mustaca et al., 2010; Núñez et al., 2015). En esta misma línea, la experiencia de una transgresión y devaluación relacional se ve influida por las evaluaciones y nivel de dolor que cada individuo percibe con dicha vivencia; sin embargo de una u otra manera siempre impactarán en el equilibrio tanto de la relación como de la víctima al vincularse con reacciones conductuales y menor satisfacción.

Además, debe tomarse en cuenta que dentro de los $\mathrm{EH}$ se han clasificado distintos tipos de violencia y transgresiones (Vangelisti y Redlick, 2017). Estas tienen una probada relación con limitaciones de las funciones físicas y psicológicas, así como con conductas negativas hacia la salud (Perales et al., 2016; Díaz et al., 2018; Méndez y García, 2015).

En lo que respecta a las mujeres con cardiopatía, resultó que a mayores sentimientos de control ante la situación en comparación con su pareja ante la experiencia de un $\mathrm{EH}$, menos problemas emocionales que las limiten a realizar actividades cotidianas y sociales (Rol emocional y función social). Ocurre lo contrario - respecto a las limitaciones mencionadas - para ambos sexos cuando se reporta mayor desconfianza y percepción de debilitamiento de la relación ante una transgresión.

Asimismo, Vangelisti y Redlick (2017) indican que, si bien, las transgresiones o 
devaluaciones en la pareja pueden impactar en diversos aspectos personales y relacionales, en ocasiones proporcionan información que posteriormente favorece el cariño, entendimiento y apoyo, con lo cual se logra un mejor control en situaciones similares. Con base en ello, dicho sentimiento de control frente a situaciones generadoras de estrés puede favorecer la satisfacción y el bienestar general del individuo e impactar positivamente en su calidad de vida (Barragán y Morales, 2014; López et al., 2014).

Respecto a la relación del amor compasivo con la calidad de vida, se observó — solamente en las mujeres - que expresar más sentimientos de apoyo, ayuda, ánimo y cuidado a la pareja, se relacionó con mayor salud reportada. Al respecto, Pietromonaco y Collins (2017) y Zepeda y Sánchez (2019) refieren que el apoyo por parte del otro ha mostrado tener influencia en diferentes aspectos relacionados con la salud en el afrontamiento y progresión de la enfermedad, así como en el ajuste y la recuperación de las diversas intervenciones a las que es sometido el paciente.

Además, el apoyo busca cumplir una función emocional mediante el confort y el cuidado que, al ser una conducta destinada al beneficio del otro, favorece la satisfacción con la relación (Solares et al., Ortiz, 2011). A su vez, esta satisfacción genera una sensación de bienestar, lo cual se vincula con la salud física y psicológica del individuo, además de otros aspectos referentes a la calidad de vida como la vitalidad y el rol emocional y la percepción de salud en general (Barragán y Morales, 2014; Granillo y Sánchez, 2020).

\section{La pareja del paciente con cardiopatía}

En relación con las parejas de los pacientes, en los hombres resultó que experimentar mayor control en comparación con la pareja ante un EH se relacionó con menores problemas emocionales que limiten la realización de actividades diarias y sociales. Asimismo, Bairero-Aguilar (2018) y García-Martínez et al. (2016) refieren que la incontrolabilidad ante situaciones estresantes o difíciles puede generar un impacto importante sobre la salud, trayendo consigo problemas físicos, dolores y limitaciones que se convierten en una fuente de enfermedad e impactan la calidad de vida de las personas al deteriorar su desempeño ideal en actividades diversas.

Además, en estos mismos participantes se observó que a mayor intención percibida de hacer daño a partir de la transgresión, mayor dolor reportado, así como menor salud y vitalidad. Aunado a esto, un EH puede vivirse de diferentes maneras en función de la intencionalidad con la que se percibió (Vangelisti y Redlick, 2017; Lemay et al., 2012). Esto resulta en que mayor intencionalidad experimentada, mayor dolor emocional ya definido como sentimientos de tristeza, ansiedad y depresión, además de inseguridad, incertidumbre ante poder ser herido nuevamente y vulnerabilidad, lo cual se ha relacionado con la salud y prevalencia de enfermedades crónicas (Agganis et al., 2010); es decir, el estado anímico parece afectar la calidad de vida siendo tanto en el componente físico como mental un predictor principal de esta (Perales et al., 2016).

Mayor autopercepción negativa se relacionó con mayores dolores corporales y 
debilitamiento. Esto ocurrió también cuando se tenía la sensación de que el evento afectó la relación al ocasionar su debilitamiento, aunado a mayores limitaciones en actividades cotidianas debido a problemas de salud. Respecto a esto, se ha encontrado que un conflicto presente en la relación de pareja, puede suponer mayor sentimiento de vulnerabilidad respecto al tema de la salud (Agganis et al., 2010; Simó et al., 2015), así como problemas para sobreponerse al impacto del EH. Esto se debe al posible estrés que supone dicha situación; se presentan diversas emociones, en este caso negativas que, al sufrir un desajuste en su intensidad debido al contexto en el que se presentan pueden resultar en afectaciones a la salud (García-Martínez et al., 2016; Montalvo-Prieto et al., 2012).

Por otro lado, en cuanto a las mujeres sanas, se observó que la percepción de que la relación se debilitó o afectó a partir de la ocurrencia de un EH se vinculó con mayores limitaciones al realizar actividades diarias y de trabajo atribuidas a problemas emocionales y mayor dolor. Además, más autopercepción negativa posterior a la transgresión se relacionó con menos vitalidad y felicidad. En congruencia con algunos resultados discutidos en apartados anteriores, las mujeres han sido reconocidas - culturalmente - con mayor facilidad para expresar y reconocer sus emociones tanto positivas como negativas en diversas situaciones (Díaz et al., 2018); ello puede resultar en vulnerabilidad psicológica que a su vez impacte su salud en mayor grado (Machín y Cifre, 2020), aunado a que dicha vulnerabilidad ha sido asociada con características de la personalidad como la autoestima y la estabilidad emocional (Molina, 2016).

Respecto a ambos sexos, los resultados indicaron que percibir mayores afectaciones personales ante el EH se relacionó con mayores limitaciones al realizar actividades cotidianas y sociales debido a problemas de tipo emocional y menor vitalidad. Esto mismo sucede cuando el efecto del evento se percibe en la relación al debilitarla y provocar desconfianza entre sus miembros. Así, la autoestima y autopercepción son aspectos relacionados con la seguridad, felicidad, satisfacción con la vida y la salud (Núñez et al., 2015; Ruiz-González et al., 2018).

De esta manera, una baja autoestima se ha vinculado con sentimientos de inseguridad y deficiencias tanto personales como patológicas (Mustaca et al., 2010; Núñez et al., 2015). Algo similar ocurre respecto a los efectos percibidos a la relación, es decir, una transgresión relacional puede impactar en mayor o menor grado el equilibrio de la relación y la víctima, al provocar menor satisfacción y desmejorar la salud de sus miembros (Perales et al., 2016; Díaz et al., 2018; Méndez y García, 2015).

Por último, en lo correspondiente al amor compasivo y la calidad de vida, resultó que solo en las mujeres sanas - estar más dispuestas a sacrificarse y preocuparse por el bienestar de su pareja se relacionó con mayor dolor corporal. Este hallazgo puede discutirse a partir de lo reportado por Díaz et al. (2018) y Machín y Cifre (2020) respecto a las atribuciones estereotípicas culturales, en las cuales se ha identificado 
a la mujer como mayormente expresiva en comparación con el hombre.

Asimismo, son ellas quienes presentan mayor vulnerabilidad psicológica, con lo que pueden verse más afectadas emocionalmente ante cualquier suceso y, con ello, sentir mayor impacto en su salud. Además, esto se aúna a la posibilidad de ser quienes cuiden de su pareja enferma, lo que resulta en sobreesfuerzos para lidiar con las demandas propias de la enfermedad, así como con el cumplimiento de sus diferentes roles dentro de la sociedad como el de ser madre, hija y empleada, los cuales pueden representar un gran carga en su día a día y un deterioro de su calidad de vida (HamamaRaz, 2010; Vaquiro y Stiepovich, 2010).

En cuanto a hombres y mujeres sanos se encontró que, a más amor altruista, mayor vitalidad y felicidad. De esta manera, la preocupación por el otro además de la búsqueda para siempre brindar apoyo y cuidado a la pareja son elementos importantes para la relación, aunados a una sensación de bienestar personaly de la relación (Sebastián, 2016) vinculada tanto a la salud física como psicológica y sentimientos de vitalidad (Bilbao et al., 2014; Zepeda y Sánchez, 2019).

\section{Conclusión}

En el presente estudio, los resultados encontrados aportan información relevante sobre la relación existente entre la experiencia de EH como variable negativa y el amor compasivo como positiva con la calidad de vida de las parejas que se enfrentan a una enfermedad crónica como los es una cardiopatía. Estos datos contribuyen a la investigación actual respecto a la experiencia de EH y sus implicaciones debido a la escasa exploración del tema en el contexto mexicano. Asimismo, se encontró que dichos eventos afectan en mayor medida a las mujeres con un padecimiento cardiaco, mientras que son los hombres en quienes resultaron mayores correlaciones respecto a la experiencia de transgresiones y deterioro de su calidad de vida en cuanto a su salud física y emocional.

Las limitaciones son respecto a que los datos aquí presentados muestran el análisis de la información obtenida en un primer momento, en el cual se ha considerado a las cardiopatías como un grupo general de enfermedad crónica; sin embargo, la diversidad de padecimientos dentro de estas cardiopatías puede incluir variaciones respecto a los síntomas, cuidados, el tiempo de diagnóstico y el tratamiento. Además, las características individuales y relacionales de la muestra como la edad de los participantes y su tiempo de relación y cohabitación con su pareja pueden resultar en diferencias referentes al grado en el que la enfermedad permea la calidad de vida de quien la padece, así como la interacción romántica.

Finalmente, la información obtenida aporta datos relevantes para las personas que atraviesan por un padecimiento, brindando apoyo $\mathrm{y}$ conocimiento a las parejas en las que, en muchas ocasiones, uno de los miembros toma el papel de cuidador y acompaña al paciente durante el proceso. Asimismo, puede ser relevante para profesionales de la salud física y psicológica, al poder considerar las variables estudiadas que, en 
conjunto con otras, puedan permear la calidad de vida de sus pacientes y con ello favorecer o no el proceso de la enfermedad, permitiendo un mejor control y seguimiento de esta.

\section{Referencias}

Agganis, B., Weiner, D., Giang, L., Scott, T., Tighiouart, H., Griffith, J. y Sarnak, M. (2010). Depression and cognitive function in maintenance hemodialysis patients. American Journal of Kidney Diseases, 56(4), 704-712. https://doi.org/10.1053/j. ajkd.2010.04.018

Aguilar Montes de Oca, Y.P., Valdez Medina, J. L., González-Arratia López-Fuentes, N. I. y González Escobar, S. (2013). Los roles de género de los hombres y las mujeres en el México contemporáneo. Enseñanza e Investigación en Psicología, 18(2), 207224. https://www.redalyc.org/articulo.oa? $\mathrm{id}=29228336001$

Álvarez Ramírez, E., García Méndez, M. y Rivera Aragón, S. (2015). Satisfacción marital a través de la dinámica del conflicto y la culpa. Psicología Iberoamericana, 23(1), 48-57. https://www.redalyc.org/articulo. oa?id=133944230006

Asociación Americana de Psicología. (2017). Principios éticos de los psicólogos y código de conducta. American Psychological Association. https://www.apa.org/ethics/ code/index.aspx.

Bachman, G. y Guerrero, L. (2006). Relational quality and communicative responses following hurtful events in dating relationships: An expectancy violations analysis. Journal of Social and Personal Relationships, 23(6), 943-963. https://doi. org/10.1177/0265407506070476

Bairero-Aguilar, M. E. (2018). El estrés y su influencia en la calidad de vida. Revista Médica Multimed, 21(6), 971-982. http:// www.revmultimed.sld.cu/index.php/mtm/ article/view/688/1054

Barragán Estrada, A. y Morales Martínez, C. (2014). Psicología de las emociones positivas: generalidades y beneficios. Enseñanza e Investigación en Psicología, 19(1), 103-118. https://www.redalyc.org/ pdf/292/29232614006.pdf

Barrantes, M. (2010). Factores asociados a la calidad de vida relacionada a la salud en pacientes con cardiopatía coronaria y diabetes mellitus. Revista Médica Herediana, 21(3), 118-127. http://www. scielo.org.pe/scielo.php?pid=S1018130X2010000300003\&script=sci_arttext

Berscheid, E. (2010). Love in the fourth dimension. Annual review of psychology, 61, 1-25. https://doi.org/10.1146/annurev. psych.093008.100318

Bilbao Cercós, A., Beniel Navarro, D., Pérez Marín, M., Montoya Castilla, I., Alcón Sáez, J. J. y Prado Gascó, V. (2014). El autoconcepto y la adaptación a la enfermedad en pacientes diabéticos pediátricos. Clínica y Salud, 25(1), 57-65. https://doi.org/10.5093/cl2014a5

Blanco, P., Ruiz-Jarabo, C., García de Vinuesa, L. y Martín-García, M. (2004). La violencia de pareja y la salud de las mujeres. Gaceta 
Sanitaria, 18(4), 182-188. http://scielo. isciii.es/scielo.php?script=sci_arttext\&pi $\mathrm{d}=$ S0213-91112004000400029

Blandón-Hincapié, A. I. y López-Serna, L.

M. (2016). Comprensiones sobre pareja en la actualidad: jóvenes en busca de estabilidad. Revista Latinoamericana de Ciencias Sociales, Niñez y Juventud, 14(1), 505-517. http://www. scielo.org.co/scielo.php?pid=S1692$715 \mathrm{X} 2016000100035 \& \mathrm{script}=\mathrm{sci}$ abstract\&tlng=pt

Burbano Rivera, D. V. (2017). Estilos y calidad de vida en salud del paciente hipertenso (Tesis inédita de licenciatura). Facultad de Enfermería, Bogotá, Colombia. https://repositorio.unal.edu.co/handle/ unal/62063

Carr, D., Freedman, V. A., Cornman, J. C. y Schwarz, N. (2014). Happy marriage, happy life? Marital quality and subjective well-being in later life. Journal of Marriage and Family, 76(5), 930-948. https://doi.org/10.1111/jomf.12133

Castañeda Hernández, A. (2015). Calidad de vida y adherencia al tratamiento de personas con enfermedad crónica oncológica. Revista Cuidarte, 6(1), 906-

913. http://dx.doi.org/10.15649/cuidarte. v6i1.146

Cozby, P. (2005). Methods in Behavioral Research. McGraw Hill.

Dalteg, T., Benzein, E., Fridlund, B. y Malm, D. (2011). Cardiac disease and its consequences on the partner relationship:
A systematic review. European Journal of Cardiovascular Nursing, 10(3), 140-149. https://doi.org/10.1016/j.ejcnurse.2011.01.006

Díaz Loving, R. y Sánchez Aragón, R. (2020). Psicología del amor: una visión integral de la relación de pareja. Miguel Ángel Porrúa/UNAM.

Díaz Loving, R., Velasco Matus, P. W. y Rivera Aragón, S. (2018). Felicidad, instrumentalidad y expresividad en dos muestras latinoamericanas: México y Bolivia. Persona, 21(1), 11-29. https:// doi.org/10.26439/persona2018.n021.1989 Díaz, M., Estévez, A., Momeñe, J. y Linares, L. (2018). Las actitudes amorosas y la satisfacción en la pareja como factores intervinientes en la relación entre la violencia y las consecuencias en la salud de las mujeres. Ansiedad y Estrés, 24(1), 31-39. https://doi.org/10.1016/j. anyes.2018.01.001

Fehr, B., Harasymchuk, C. y Sprecher, S. (2014). Compassionate love in romantic relationships: A review and some new findings. Journal of Social and Personal Relationships, 31(5), 575-600. https://doi. org/10.1177/0265407514533768

Flórez Alarcón, L., Mercedes Botero, M. y Moreno Jiménez, B. (2005). Psicología de la salud. Temas actuales de investigación en Latinoamérica. Alapsa.

Flórez Rodríguez, N. y Sánchez Aragón, R. (2019). Evaluando el amor compasivo en la pareja. Vertientes: Revista Especializada 
en Ciencias de la Salud, número especial, 55-57. https://www.zaragoza.unam.mx/3congreso-internacional-de-psicologia-dela-fes-zaragoza/

Granillo Velasco, L. F. y Sánchez Aragón, R. (2020). Deteriorando la salud en pareja: el papel de los eventos hirientes. Enseñanza e Investigación en Psicología, 2(2), 203215. https://revistacneip.org/index.php/ cneip/article/view/107

Gumán, J., Treviño, R. y Cámara, A. D. (2015). Posición en el hogar y género. Desigualdades en la calidad de vida relacionada con la salud entre la población adulta en España. Revista Internacional de Sociología, 73(1), 03-17. https://doi. org/10.3989/2013.03.04

Hamama-Raz, Y. (2010). Does psychological adjustment of melanoma survivors differ between genders? Psycho-Oncology, 21(3), 255-263. https://doi.org/10.1002/ pon. 1889

Hernández Sampieri, R., Fernández Collado, C. y Baptista Lucio, M. (2014). Metodología de la Investigación. McGraw Hill.

Jasso-Soto, M. E., Pozos-Magaña, M. G., Cadena-Estrada, J. C. y Olvera-Arreola, S. S. (2017). Calidad de vida y perspectiva espiritual de los pacientes hospitalizados con enfermedad cardiovascular. Revista de Enfermería del Instituto Mexicano del Seguro Social, 25(1), 9-17. http:// revistaenfermeria.imss.gob.mx/editorial/ index.php/revista_enfermeria/article/ view/84/308
Leary, M. R. (2015). Emotional responses to interpersonal rejection. Dialogues in Clinical Neuroscience, 17(4), 435 - 441 .https://www.ncbi.nlm.nih. gov/pmc/articles/PMC4734881/pdf/ DialoguesClinNeurosci-17-435.pdf Ledón Llanes, L. (2011). Enfermedades crónicas y vida cotidiana. Revista Cubana de Salud Pública, 37(4), 488- 499. http://scielo. sld.cu/scielo.php?script=sci_arttext\&pid $=$ S0864-34662011000400013

Lemay, E. P., Overall, N. C. y Clark, M. S. (2012). Experiences and interpersonal consequences of hurt feelings and anger. Journal of personality and social psychology, 103(6), 982. https://doi. org/10.1037/a0030064

López Santana, Y., Díaz Berasategui, Y., Cintra Hernández, Y. y Limonta Rodriguez, R. (2014). Estrés, el "gran depredador". Revista Información Científica, 84(2), 375-384. https://www.redalyc.org/ articulo.oa? $\mathrm{id}=551757261019$

Machin Rincón, L. y Cifre Gallego, E. (2020). La variabilidad de los rasgos estereotípicos de género: un estudio de diario. Agora de Salut, 7, 155-165. http:// dx.doi.org/10.6035

Mao-Chia, S. (2017). The positive side of hurtful communication: when hurt feelings improve close relationships (Tesis inédita de licenciatura). The University of Texas at Austin, Austin, Texas. https://repositories. lib.utexas.edu/handle/2152/63637 
Martínez-Álvarez, J. L., Fuertes-Martín, A., Orgaz-Baz, B., Vicario-Molina, I. y González-Ortega, E. (2014). Vínculos afectivos en la infancia y calidad en las relaciones de pareja de jóvenes adultos: el efecto mediador del apego actual. Anales de Psicología, 30(1), 211-220. https://doi. org/10.6018/analesps.30.1.135051

Maureira Cid, F. (2011). Los cuatro componentes de la relación de pareja. Revista electrónica de psicología Iztacala, 14(1), 321-332. https://www.revistas.unam.mx/index.php/ repi

McCoy, A., Rauer, A. y Sabey, A. (2017). The Meta marriage: links between older Couples' relationship narratives and marital satisfaction. Family process, 56(4), 900-914. https://doi.org/10.1111/ famp. 12217

Méndez Sánchez, M. P. y García Méndez, M. (2015). Relación Entre las Estrategias de Manejo del Conflicto y la Percepción de la Violencia Situacional en la Pareja. Revista Colombiana de Psicología, 24(1), 99-111. https://www.redalyc.org/articulo. oa? id=80438019007

Molina Rodríguez, A. (2016). Vulnerabilidad y daño psíquico en mujeres víctimas de violencia en el medio familiar (Tesis inédita de licenciatura). Universidad de Granada, Granada, España. https:// digibug.ugr.es/handle/10481/43550

Montalvo-Prieto, A., Cabrera-Nanclares, B. y Quiñones-Arrieta, S. (2012). Enfermedad crónica y sufrimiento: revisión de literatura. Aquichan, 12(2), 134-143. https:// www.redalyc.org/ articulo. oa?id=74124103005

Moreno, B., Garrosa, E. y Gálvez, M. (2005). Personalidad positiva y salud. En L. Flórez Alarcón, M. Mercedes Botero y B. Moreno Jiménez (Eds.), Psicología de la salud. Temas actuales de investigación en Latinoamérica (pp. 59-76). Alapsa. https://www.academia.edu/9178674/ PERSONALIDAD_POSITI VA_Y_ SALUD

Mustaca, A., Kamenetzky, G. y Vera., P. (2010). Relaciones entre Variables Positivas y Negativas en una Muestra de Estudiantes Argentinos. Revista Argentina de Clínica Psicológica, 19(3), 227-235. http://hdl. handle.net/11336/15103

Núñez, M., González, G. y Realpozo, R. (2015). Relación entre autoestima y felicidad desde la psicología positiva en estudiantes de enfermería intercultural. Enfermería Actual en Costa Rica, 29, 1-18. http:// dx.doi.org/10.15517/revenf.v0i29.19726

Perales, C., Duschek, S. y del Paso, G. (2016). Calidad de vida relacionada con la salud en la enfermedad renal crónica: relevancia predictiva del estado de ánimo y la sintomatología somática. Nefrología, 36(3), 275-282. https://doi.org/10.1016/j. nefro.2015.12.002

Pérez Pérez, A. E. y Sánchez Aragón, R. (2016). Eventos hirientes en la pareja desde la perspectiva de la víctima. En R. Díaz Loving, I. Reyes Lagunes, S. 
Rivera Aragón, J. E. Hernández Sánchez y R. García Falconi (Eds.), Aportaciones Actuales de la Psicología Social (pp. 7176). Asociación Mexicana de Psicología Social.

Pietromonaco, P. R. y Collins, N. L. (2017). Interpersonal mechanisms linking close relationships to health. American Psychologist, 72(6), 531. https://doi. org/10.1037/amp0000129

Ruiz-González, P., Medina-Mesa, Y., Zayas, A. y Gómez-Molinero, R. (2018). Relación entre la autoestima y la satisfacción con la vida en una muestra de estudiantes universitarios. Revista INFAD de Psicología. International Journal of Developmental and Educational Psychology, 2(1), 67-76. http://www. infad.eu/RevistaINFAD/OJS/index.php/ IJODAEP/article/view/1170

Sabey, A. K., Rauer, A. J. y Haselschwerdt, M. L. (2014). "It's not just words coming from the mouth" The nature of compassionate love among older couples. Journal of Social and Personal Relationships, 33(5), 640-665. https://doi. org/10.1177/0265407515588219

Sánchez Aragón, R. (2018). Afectividad y coregulación en la pareja: validación de un inventario y cambios a través del ciclo vital. Revista Psicologia e Educação On-Line, 1(1), 58-6. http:// psicologiaeeducacao.ubi.pt/Files/Other/ Artigos\%20OnLine/2018/V1N1\%20 online/6.\%20V1N1online2018.pdf
SánchezAragón, R., García Meraz, M. y Martínez Trujillo, B. (2017). Encuesta de Salud SF36: validación en tres contextos culturales de México. Revista Iberoamericana de Diagnóstico y Evaluación Psicológica, 3(45), 5-16. https://www.redalyc.org/ articulo.oa?id=459653862002

Sebastián Pabón, M. (2016). Relación de pareja en la unión libre-Estudio de caso. Ajayu Órgano de Difusión Científica del Departamento de Psicología, 14(2), 371394. http://www.scielo.org.bo/scielo. php?script=sci_arttext\&pid=S2077$21612016000200008 \& \operatorname{lng}=\mathrm{es} \& \mathrm{nrm}=\mathrm{iso}$ Simó Noguera, C., Hernández Monleón, A., Muñoz Rodríguez, D. y González Sanjuan, M. (2015). El efecto del estado civil y de la convivencia en pareja en la salud. Revista Española de Investigaciones Sociológicas, 151(1), 141-165. https:// doi.org/10.5477/cis/reis.151.141

Sociedad Mexicana de Psicología. (2010). Código ético del psicólogo. Trillas.

Solares, S., Benavides, J., Peña, B., Rangel, D. y Ortiz, A. (2011). Relación entre el tipo de apoyo y el estilo de amor en parejas. Enseñanza e investigación en psicología, 16(1), 41-56. https://www.redalyc.org/ articulo.oa? $\mathrm{id}=29215963004$

Suárez Acuña, C. y Monroy Garzón, A. (2012). Afrontamiento y adaptación de los padres durante el postoperatorio de cardiopatía congénita. Index de Enfermería, 21(3), 145-149. http://dx.doi.org/10.4321/S1132 $-12962012000200008$ 
Urzúa, A. (2010). Calidad de vida relacionada con la salud: elementos conceptuales. Revista médica de Chile, 138(3), 358-365. http://dx.doi.org/10.4067/S0034-9887 2010000300017

Urzúa, A. y Caqueo-Urízar, A. (2012). Calidad de vida: una revisión teórica del concepto. Terapia psicológica, 30(1), 6171. http://dx.doi.org/10.4067/S071848082012000100006

Vangelisti, A. L. y Redlick, M. (2017). Hurt Feelings. En J. Fitzgerald (Ed.), Foundations for couples' therapy: Research for the real world (p. 186-196). Routledge/Taylor \& Francis Group. https://doi.org/10.4324/978131567861019

Vaquiro Rodríguez, S. y Stiepovich Bertoni, J. (2010). Cuidado informal, un reto asumido por la mujer. Ciencia y enfermería, 16(2), 17-24. http://dx.doi.org/10.4067/S071795532010000200002

Vinaccia-Alpi, S. y Quiceno, J. (2012). Calidad de vida relacionada con la salud y enfermedad crónica: estudios colombianos. Psychologia, 6(1), 123-136. https://doi.org/10.21500/19002386.1175

Ware, J. E. y Sherbourne, C. D. (1992). The MOS 36-item short-form health survey (SF-36): I. Conceptual framework and item selection. Medical Care, 30(6), 473483.https://www.jstor.org/stable/3765916 Zepeda Goncen, G. D. y Sánchez Aragón, R. (2019). Efectos del apego, afecto y capacidad de recibir apoyo en la salud de la pareja. Revista Psicologia $e$ Educação On-Line, 2(1), 64-74. http:// psicologiaeeducacao.ubi.pt/Files/Other/ Artigos\%20OnLine/2019V1/8-\%20 V2N1online2019.pdf

Enviado: 05 de abril de 2020

Revisado: 01 de agosto de 2020

Aceptado: 04 de octubre de 2020 